WellBeing International

WBI Studies Repository

1989

\title{
Conspecific Recognition in the Chimpanzee (Pan troglodytes): Cardiac Responses to Significant Others
}

Sarah T. Boysen

The Ohio State University

Gary G. Berntson

Emory University

Follow this and additional works at: https://www.wellbeingintlstudiesrepository.org/acwp_asie

Part of the Animal Studies Commons, Comparative Psychology Commons, and the Other Animal Sciences Commons

\section{Recommended Citation}

Boysen, S. T., \& Berntson, G. G. (1989). Conspecific recognition in the chimpanzee (Pan troglodytes): cardiac responses to significant others. Journal of Comparative Psychology, 103(3), 215.

This material is brought to you for free and open access by WellBeing International. It has been accepted for inclusion by an authorized administrator of the WBI Studies Repository. For more information, please contact wbisr-info@wellbeingintl.org.

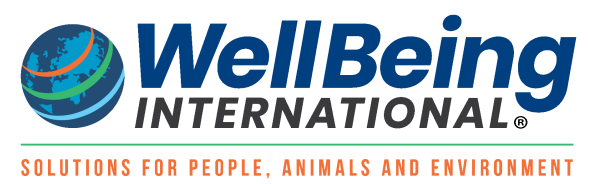




\title{
Conspecific Recognition in the Chimpanzee (Pan troglodytes): Cardiac Responses to Significant Others
}

\author{
Sarah T. Boysen and Gary G. Berntson \\ Ohio State University and Emory University
}

\begin{abstract}
$\underline{\text { ABSTRACT }}$
The ability of a chimpanzee (Pan troglodytes) to recognize photographs of conspecifics was evaluated with heart-rate measures. Heart rate was recorded before, during, and after viewing photographs of an aggressive chimpanzee, a friendly companion animal, and an unfamiliar chimpanzee. The subject displayed a differential pattern of heart-rate response to the stimulus animals, without prior experience with the photographs. Responses to the aggressive animal were acceleratory, which suggests a defensive response. Respiratory sinus arrhythmia suggested that this response was associated with sympathetic activation. In contrast, responses to the familiar animal were minimal, whereas cardiac deceleration was observed in response to the strange chimp, which likely reflects an orienting response. Results suggest the chimpanzee is able to recognize photographs of individual conspecifics and that heart-rate change can reflect the nature of established social relationships between chimpanzees.
\end{abstract}

The capacity to recognize individual conspecifics confers a significant adaptive advantage, particularly for those species that depend on rank, social hierarchy, or kinship for status within a group (e.g., Smuts, Cheney, Wrangham, \& Struhsaker, 1987). Such dynamic relationships are observed among various primate species, and thus the demand may often arise for rapid discrimination and recognition of and response to a specifc animal in a contextually appropriate manner (e.g., Cheney \& Seyfarth, 1986; Gouzoules, Gouzoules \& Marler, 1984). Studies with captive primates have also indicated a range of capabilities among monkeys and apes, in relation to conspecific preference or recognition (Hamilton, 1977; Overman \& Doty, 1982; Rosenfeld \& Van Hoesen, 1979; Sackett, 1965, 1966; Sackett, Griffin, Pratt, Josyln \& Ruppenthal, 1967). Sackett et al. reported that monkeys preferred to view conspecifics with whom they had established a social relationship. In a study of face recognition in rhesus macaques, Rosenfeld and Van Hoesen found that once monkeys learned a full-face discrimination of a photograph of an unfamiliar monkey, they readily acquired additional discriminations in which a variety of stimulus characteristics were altered. Those authors suggested that once the initial facial discriminations were made, the monkeys were able to use a range of cues in responding to rather dramatic changes in other stimulus dimensions (Rosenfeld \& Van Hoesen, 1979). They also suggested that the facial stimuli were perceived as other monkeys, because the animals rapidly retreated to the rear of the testing apparatus and threatened novel facial photographs.

Sackett (1966) explored the effects of visually presented social and nonsocial stimulation on monkeys who had been reared in social isolation. Photographs of infant monkeys and threatening animals produced the greatest overall behavioral responses, and pictures of threatening animals evoked more 
disturbance behaviors (such as rocking, self-clasping, and withdrawal) than did pictures of infants (Sackett, 1966). He concluded that such socially meaningful stimuli may have unlearned, prepotent properties for socially naive monkeys, and in particular, photographs of threat behavior appeared to function as a releasing stimulus for fearful behavior in young monkeys. Sackett concluded that there may exist species-specific, innate recognition mechanisms in primates.

In a study of hemispheric specialization in macaques and humans, Overman and Doty (1982) reported that monkeys who encountered novel images of monkeys and humans differentiated them from other classes of visual stimuli: The monkeys exhibited three times the number of species-typical responses for the faces compared with naturalistic stimuli such as flowers or landscapes. These animals also treated inverted face stimuli differently than they did other inverted photographs. These findings provide additional evidence that the monkeys recognized photographs of other monkeys and humans and responded appropriately (Overman \& Doty, 1982).

Primates, therefore, appear able to learn discriminations among individual photographs and to respond differentially to novel facial photographs. What remains less clear, however, is the extent to which nonhuman primates are able to recognize the specific animals depicted in photographic representations. Less experimental evidence is available for the great apes, although Gallup (1970) demonstrated that chimpanzees could differentiate mirror images of themselves from images of conspecifics. The critical stimulus features and the nature of the intervening perceptual representations that support these discriminative skills, however, are unclear. Tentative evidence suggests that movement, or movementrelated feedback in the case of the mirror studies, may not be crucial in visual recognition. Bauer and Philip (1983) reported that chimpanzees could distinguish among animals from static photographic representations, although their study included an initial regimen of discrimination training with the photographs. Thus, although it appears that discrimination was achieved, it remains uncertain if the established perceptual representations entailed an explicit recognition of the individual animals in the photographs.

We recently demonstrated through the use of heart-rate measures that a chimpanzee could recognize photographic representations of individual humans with whom she shared a strong social relationship (Boysen \& Berntson, 1986). This was evidenced by a large and significant cardiac deceleratory orienting response that appeared selectively on presentation of slide photographs of persons who served as the animals' principal caregivers. Importantly, this recognition was manifest in the absence of prior training or experience with the photographs. In contrast, slides of two additional categories of humans, strangers and familiar persons who had not directly interacted with the test animal, failed to evoke appreciable heart-rate responses. These results indicate that the chimpanzee is able to recognize photographic depictions of individual humans in the absence of specific discrimination training. They further suggest that heart rate may provide a quantitative index of the distinct social relationships among animals and may offer an important tool for studies of conspecific recognition in primates.

We further examine these issues in this study by measuring the heart-rate responses of a juvenile chimpanzee (Sheba) to novel photographs of conspecifics with whom she had differing social relationships. It was hypothesized that photographic representations of an animal who was typically aggressive toward Sheba would elicit cardiac acceleration, indicating a defensive or fearlike response (Graham, 1984; Sokolov, 1963). It was further hypothesized that cardiac responses to slides of an animal with whom Sheba shared positive social interactions would be minimal or deceleratory. Finally, responses to a third category of slides that depicted an unfamiliar chimpanzee were also expected to be deceleratory, which would reflect a cardiac orienting response to the novelty of the strange animal (Graham, 1984; Siddle, 1983). 


\section{Method}

Subject

A 4.5-year-old female chimpanzee (Pan troglodytes), Sheba, served as the subject. Sheba was separated from her biological mother at 4 months and human cross-fostered until 2 years of age. At that time she joined the Primate Cognition Project. During the course of this project, Sheba established a positive social interaction with a young male chimpanzee, Kermit (5.5 years old). Another male, Darrell (6 years old), was physically larger than the other animals and consistently consistently responded aggressively toward Sheba. The three animals could not be left together unattended because of the intensity of Darrell's responses toward Sheba. Sheba and Kermit, however, continued to display ageappropriate, gregarious social interactions. Moreover, Kermit often defended Sheba from Darrell's attacks, which frequently resulted in Kermit's being the target of a redirected attack. During the course of the study, however, Sheba had no interactions with other animals in the project.

Since her arrival in our laboratory, Sheba spent approximately 4-6 hr per day interacting with human teachers and learning a variety of conceptual skills. None of the training, however, entailed the explicit recognition of, or responding to, specific animals or photographs of animals.

\section{Electrocardiographic (ECG) Measures}

The ECG signal was measured by disposable silver/silver chloride electrodes attached to standard thoracic monitor sites and secured with an elastic bandage. The ECG electrodes were connected to an Amerec ERM 101 cardiotachometer with the R-wave-detector output coupled to a microcomputer for online determination of discrete heart periods. The raw ECG signal was also recorded on FM tape (Cardiodyne Cardiocassette) for off-line playback. Potential artifacts in the heart-period record were identified off line by the computer system as heart periods that deviated from surrounding beats by more than $35 \%$. Heart-period values that exceeded this criterion were verified or corrected by direct measurement from the polygraph record. In addition to heart period data, second-by-second heart-rate values were derived for $4 \mathrm{~s}$ before and $12 \mathrm{~s}$ after each stimulus. Heartrate measures were based on a proportionately weighted average of beats falling wholly or partially within a given second. Averaged second-by-second heart-period values were then converted to rate.

\section{Procedure}

We measured Sheba's cardiac responses to slide photographs of chimpanzee faces. Photographs of three male juvenile chimpanzees and blank control slides were repeatedly presented to Sheba over 12 experimental sessions. Two sessions were given each day, and the sessions were separated by $30 \mathrm{~min}$. The slide stimuli consisted of color facial photographs of two chimpanzees from the project (Kermit and Darrell) and an additional unfamiliar chimpanzee. Image size, intensity, and background were equated. The stimuli were projected on a screen approximately $2 \mathrm{~m}$ from the subject $\left(20^{\circ}\right.$ visual angle; $40 \mathrm{~lx}$; background, $10 \mathrm{~lx}$ ). Each slide was presented for $8 \mathrm{~s}$, and heart rate measures were obtained before, during, and after each stimulus presentation.

All testing was completed with the animal in a quiet alert state. During testing Sheba was not restrained; she sat with an experimenter who was unaware of the slide sequence and who was unable to see the projected images. During each of the 12 sessions, six facial photographs, two different slides of each of the three target animals, were presented in block-randomized sequence. In addition, two blank control slides were presented before, and one presented after, the slide sequence in each session. Intertrial intervals averaged $30 \mathrm{~s}$ (25-35 s, randomized). In a few cases, however, testing was suspended for a 
brief period (less than $5 \mathrm{~min}$ ) when the animal became active or when prestimulus heart rate deviated from baseline values by more than 10 beats per min.

\section{Data Analysis}

Because cardiac responses were generally monophasic, overall analyses were based on the change in heart rate from the 4-s prestimulus baseline to the 8-s periods during which the slide stimuli were presented. To minimize the effect of novelty related to the first slide presentation (always a blank control slide), the results from the first trial were excluded from analysis. This resulted in a total of two stimulus presentations per session of each slide category (aggressor, companion, stranger, and control). Friedman's analysis of variance was used to evaluate overall differences among stimulus conditions, and post hoc comparisons were made with the Wilcoxon test.

In addition to heart-rate changes, heart-period variability was also evaluated during stimulus presentations by the successive difference mean square statistic (Heslegrave, Ogilvie \& Furedy, 1979). This index was used because it is minimally contaminated by phasic changes in heart-rate level (Heslegrave et al., 1979). Because heart-period variability arises from several sources, beat-by-beat data in the frequency domain was also analyzed to identify specific frequency components of this variability. The most prominent of these is generally a periodic fluctuation associated with the respiratory cycle (Porges, McCabe, \& Yongue, 1982). Termed respiratory sinus arrhythmia (RSA), this cyclic variation is attributable to reflex alterations in vagal tone, and its magnitude has been shown to be highly indicative of the level of vagal activity (Porges et al., 1982). in this experiment an index of RSA was derived according to the general methods outlined by Porges and colleagues (McCabe, Yongue, Ackles \& Porges, 1985; Porges et al., 1982). In brief, heart-period values were derived at fixed intervals (250 ms) for the epochs that extended from $1 \mathrm{~s}$ before to $15 \mathrm{~s}$ after each stimulus. These values were obtained by a weighted mean of discrete heart periods that fell wholly or partially within each interval. Trial-by-trial data were then high pass filtered (at $0.06 \mathrm{~Hz}$ ) by a third-degree polynomial. This served to minimize contamination from slow phasic heart-rate responses to the stimuli and to achieve stationarity of the time series (constant mean and variance), as is required for spectral analysis. The data were then tapered with a modified Hanning window and submitted to a discrete Fourier transform (DFT) by using the Cooley-Tukey algorithm (Cooley \& Tukey, 1965). An index of RSA was derived as the integral area under the DFT function for the frequency values from 0.1 to $0.5 \mathrm{~Hz}$. This range has been used previously with animals (McCabe et al., 1985) and encompassed the respiratory frequencies of our subject. These data were evaluated by Friedman's test.

\section{Results}

Heart-rate responses to blank control slides and slides of the unfamiliar and companion chimpanzees were predominantly deceleratory or negligible. In contrast, slides of the aggressive animal evoked notable cardiac acceleration (Figure 1). Baseline heart rate during the prestimulus periods did not differ significantly across experimental categories (prestimulus heart rate on trials with blank control slides $=$ $93.0 \mathrm{bpm}$, with the aggressor $=91.0 \mathrm{bpm}$, with the companion $=92.7 \mathrm{bpm}$, and with the stranger $=95.7$ bpm, Friedman's $X^{2}(3, N=12)=5.10$, ns. Significant differences, however, emerged across slide categories in the poststimulus heart-rate responses, Friedman's $X^{2}(3, N=12)=13.90, p<.005$. Further analyses revealed that these differences were attributable to the significantly higher heart rates during slide presentations of the aggressive animal in relation to all other stimulus categories, Wilcoxon $\operatorname{Ts}(N=$ $12)<11, p s<.03$. The mean change in heart rate during slide presentations of the aggressor animal was 5.4 ( $\pm 1.4 \mathrm{SE}$ ) bpm, compared with $-2.2( \pm 2.0) \mathrm{bpm}$ for blank slides, 0.3 ( \pm 1.5 ) bpm for companion slides, and $-3.4(+1.7) \mathrm{bpm}$ to slides of the unfamiliar chimpanzee. The cardioacceleratory response to the aggressor animal was observed consistently over sessions and showed no apparent habituation. 
Other comparisons revealed that responses to the unfamiliar animal were significantly more deceleratory than those to the companion animal, Wilcoxon $T(N=12)=13, p<.05$, whereas responses to control slides were intermediate and did not differ significantly from either the companion or stranger categories.

Figure 1. Heart-rate (HR) responses in beats per min (BPM) of a chimpanzee to slide photographs of three conspecifics with differing social relations to the test animal and to a blank control slide. (The time of stimulus onset and offset are illustrated by the arrows on the abscissa. Baseline $=$ prestimulus period.)

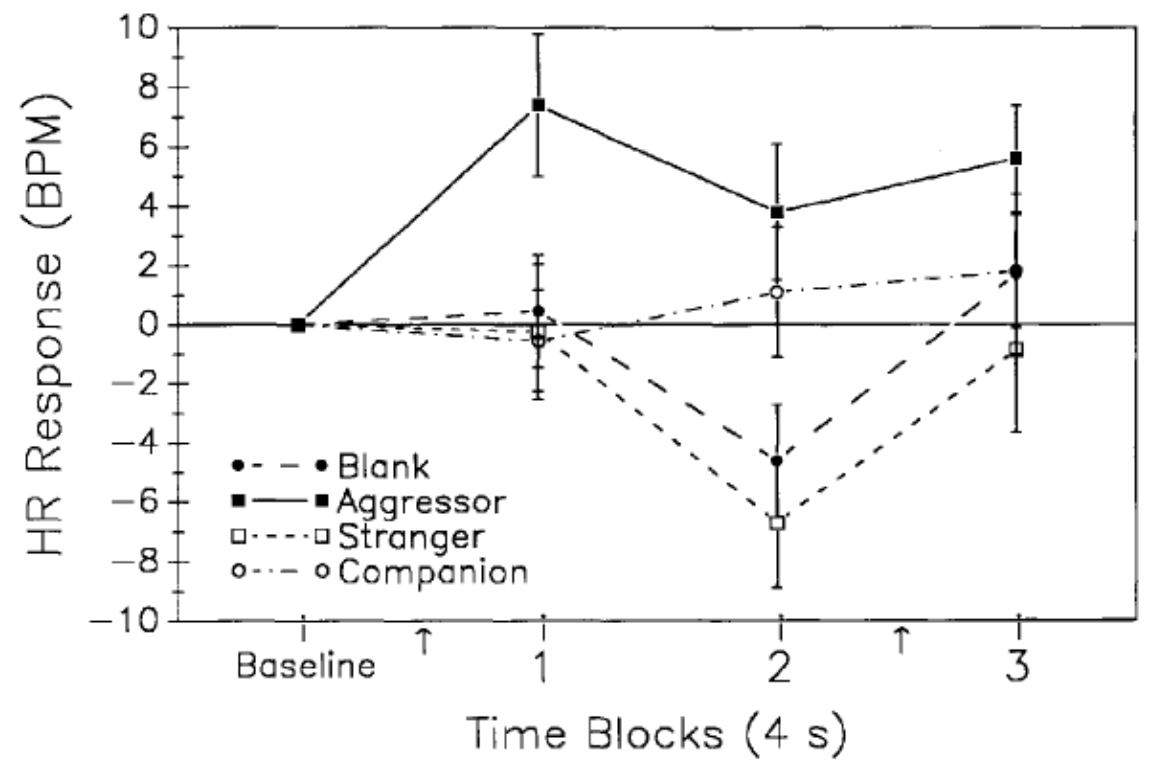

Figure 2. Spectral analysis of heart period variability, which illustrates the magnitude of respiratory sinus arrhythmia (RSA) of a chimpanzee during slide presentations of different categories of conspecifics. (Vertical lines illustrate the frequency band from which the index of RSA was derived.)

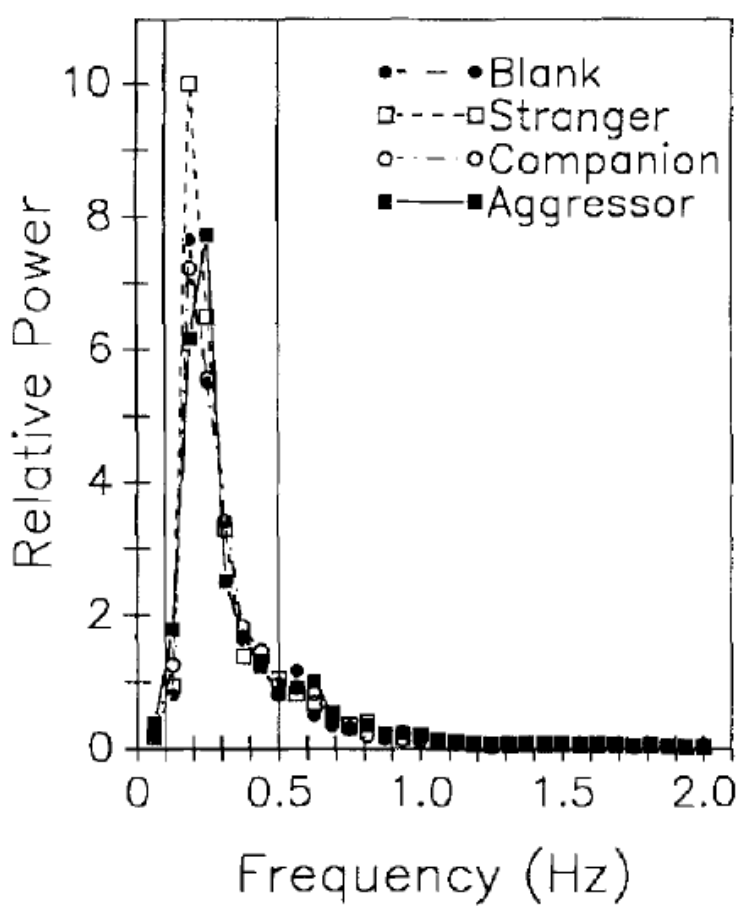


The cardioacceleratory responses to the aggressor slide were not associated with any observable somatic response. On the initial presentation of the slide of the unfamiliar chimpanzee, however, Sheba displayed a smilelike facial expression that recurred on four subsequent occasions. Smiling was not observed during presentation of any other slide category, nor were other somatic responses manifest during testing.

In spite of differences in the magnitude and direction of heart-rate change, overall heart-period variability, as indexed by the successive difference mean square statistic (Heslegrave et al., 1979), did not differ significantly among the stimulus categories. Neither were differences apparent in the magnitude of RSA, a component of heart period variability that is highly indicative of the level of vagal activity (Porges et al., 1982). A DFT was used to analyze the heart-period data in the frequency domain, and the integral spectral power in the respiratory band $(0.1-0.5 \mathrm{~Hz}$ ) was taken as an index of RSA magnitude (McCabe et at., 1985; Porges et al., 1982). The results are illustrated in Figure 2. Although the RSA magnitude associated with the cardiac deceleration to the unfamiliar animal was somewhat larger than that associated with other slide categories, the differences among stimuli failed to achieve significance.

\section{Discussion}

The distinctive patterns of evoked cardiac response to slides of different social categories of animals indicate that Sheba was able to recognize individual conspecifics depicted in the photographs. The results can also be used to suggest that the direction of heart-rate response may provide a qualitative index that reflects the nature of the social relationship between the test animal and the pictured chimpanzee. These data are consistent with a previous report of differential heart-rate responses of a chimpanzee to photographs of human faces (Boysen \& Berntson, 1986) and with reports that autonomic measures, including heart rate and pupillary and electrodermal responses, can reflect recognition of specific persons by human infants (Banks \& Wolfson, 1967; Fitzgerald, 1968; Tranel, Fowles, \& Damasio, 1985).

In our study a history of aggressive interactions between Sheba and Darrell was expressed in cardiac acceleratory responses to photographs of the aggressive animal. In contrast, slides of other chimpanzees evoked negligible or decelerator responses. These differences cannot be accounted for by simple physical features of the stimuli, because the slides were equated for retinal size, photic intensity, and general composition. Rather, the differential patterns of evoked response appear to be attributable to the social significance of the specific animals depicted. Our results do not permit a dissociation of the role of prior experience from potential morphological, archetypal features of the aggressive animal that may have contributed to the response. We have previously demonstrated, however, that Sheba displays differential responses to photographs on the basis of her social interactions with the persons presented. Thus, it is clear that social learning significantly impacts on the pattern of cardiac response.

The acceleratory heart-rate responses to the photographs of the aggressive animal likely reflected the cardiac correlate of the defensive response. The defensive response is typically observed in response to intense or aversive stimuli and is associated with minimally habituating cardiac acceleration in humans (Graham, 1979, 1984; Turpin, 1983) and apes (Berntson \& Boysen, 1989a, 1989b). Consistent with this suggestion is the similarity between the acceleration observed in response to the aggressive animal in our study and previously reported cardioacceleratory responses of chimpanzees to fear-provoking stimuli or conspecific threat barks (Berntson \& Boysen, 1989a, 1989b).

The defensive response is generally considered to arise from sympathoadrenal activation (Turpin, 1986). Cardiac acceleration, however, can result from either an increase in sympathetic activity or a decrease in vagal activity. Measures of RSA are consistent with a sympathetic origin of the cardiac acceleration in this 
study. Porges et al. (1982) demonstrated that the magnitude of RSA offers a reliable index of vagal activity, and we previously observed the expected increase in RSA amplitude during deceleratory cardiac orienting responses in the chimpanzee (Berntson \& Boysen, 1989a). In contrast, no differences in RSA amplitude during stimulus presentations in this study were apparent between the blank control slides, which yielded cardiac deceleration, and the slides of the aggressor, which were associated with cardiac acceleration. This observation is consistent with a sympathetic basis for the cardioacceleratory response and argues against an explanation in terms of vagal inhibition. Nevertheless, a decrease in vagal activity might still have been expected, given the typical reciprocity between sympathetic and parasympathetic systems. It is now clear, however, that autonomic reciprocity is subject to breakdown under certain circumstances. This is especially true during conditions of stress or other states of notable sympathetic activation, in which vagal activity may continue unabated (Grossman, 1983; Koizumi, Terui, \& Kollai, 1983). We previously observed a similar pattern of cardiac acceleration, without a corresponding decrease of vagal tone, in response to conspecific threat barks in chimpanzees (Berntson \& Boysen, 1989b).

Alternatively, it is possible that the RSA index is simply not sensitive enough to detect phasic changes in vagal activity. We previously found, however, that the magnitude of RSA is highly sensitive to the deceleratory cardiac responses evoked by photographic slides of human caregivers, under experimental conditions comparable to those of this study (Berntson \& Boysen, 1989a). In view of these considerations, our results are most consistent with the view that slides of the aggressor animal evoked a cardiac defensive-like response associated with sympathetic activation. Although the relative involvement of vagal and sympathetic systems will require further direct confirmation, the fact remains that a pattern of cardiac acceleration uniquely characterized the response to photographic depictions of an aggressive animal.

The contrasting deceleratory heart-rate responses observed to the blank slide and the photograph of the unfamiliar animal likely denote orienting responses. The orienting response has previously been found to be associated with cardiac deceleration in humans (Graham, 1979, 1984), monkeys (Weisbard \& Graham, 1971), and apes (Berntson \& Boysen, 1984). Heart-rate responses to the familiar animal were negligible, however, and differed significantly from the deceleratory responses to the unfamiliar chimpanzee. This difference may have been attributable to the greater novelty or other features of the strange animal: Slides of this animal appeared to evoke considerable interest by Sheba. This was evidenced by smile-like responses that were seen on 5 of the 24 presentations of the unfamiliar animal. Such responses were never manifest with any other stimulus slide.

In summary, it is clear that the evoked cardiac response to slides of conspecifics can significantly differentiate among animals that have different social relations with the test animal. Furthermore, it appears that distinct patterns of cardiac response may reflect the qualitative features of these relationships. Although the generalizability of these findings is limited by the single-subject design, the results indicate that Sheba, in the absence of explicit training or task demands, was able to recognize individual chimpanzees from photographic depictions. Moreover, the perceptual representations engendered by these photographs appeared to entail components of the affective response to the conspecifics depicted.

\section{References}

Banks, J. H., \& Wolfson, J. H. (1967, April). Differential cardiac response of infants to mother and stranger. Paper presented at the meeting of the Eastern Psychological Association, Boston.

Bauer, H. R., \& Philip, M. M. (1983). Facial and vocal individual recognition in the common chimpanzee. Psychological Record, 33, 161-170. 
Berntson, G. G., \& Boysen, S. T. (1984). Cardiac startle and orienting responses in the great apes. Behavioral Neuroscience, 98, 914-918.

Berntson, G. G., \& Boysen, S. T. (1989a). Cardiac correlates of cognition in infants, children and chimpanzees. In L. P. Lipsitt \& C. Rovee-Collier (Eds.), Advances in infancy research (pp. 187220). New York: Ablex.

Berntson, G. G., \& Boysen, S. T. (1989b). Specificity of the cardiac response to conspeciflc vocalizations in chimpanzees. Behavioral Neuroscience, 103, 235-245.

Boysen, S. T., \& Berntson, G. G. (1986). Cardiac correlates of recognition in the chimpanzee (Pan troglodytes). Journal of Comparative Psychology, 100, 312-324.

Cheney, D. L., \& Seyfarth, R. M. (1986). The recognition of social alliances by vervet monkeys. Animal Behavior, 34, 1722-1731.

Cooley, J. W., \& Tukey, J. W (1965). An algorithm for the machine computation of complex Fourier Series. Mathematical Computing, 19, 297-301.

Fitzgerald, H. E. (1968). Autonomic pupillary reflex activity during early infancy and its relation to social and nonsocial stimuli. Journal of Experimental Child Psychology, 6, 470-482.

Gallup, G. G., Jr. (1970). Chimpanzees: Self-recognition. Science, 167, 86-87.

Gouzoules, S., Gouzoules, H., \& Marler, P. (1984). Rhesus monkey (Macaca mulatta) screams: Representational signalling in the recruitment of agonistic aid. Animal Behavior, 32, 182-193.

Graham, F. K. (1979). Distinguishing among orienting, defensive, and startle reflexes. In H. D. Kimmel, E. H. van Olst, \& J. F. Orelbeke (Eds.), The orienting reflex in humans (pp. 137-167). Hillsdale, N J: Erlbaum.

Graham, F. K. (1984). An affair of the heart. In M. G. H. Coles, J. R. Jennings, \& J. A. Stern (Eds.), Festschrift for Beatrice and John Lacey (pp. 171-187). New York: Van Nostrand Reinhold.

Grossman, P. (1983). Respiration, stress and cardiovascular function. Psychophysiology, 20, 284-300.

Hamilton, C. R. (1977). An assessment of hemispheric specialization in monkeys. In S. J. Dimond \& D. A, Blizard (Eds.). Annals of the New York Academy of Sciences: Vol. 299. Evolution and lateralization of the brain (pp. 222-232). New York: New York Academy of Sciences.

Heslegrave, R. J., Ogilvie, J. C., \& Furedy, J. J. (1979). Measuring baseline-treatment differences in heart rate variability: Variance versus successive difference mean square and beats per minute versus interbeat intervals. Psychophysiology, 16, 151-157.

Koizumi, K., Terui, N., \& Kollai, M. (1983). Neural control of the heart. Significance of double innervation re-examined. Journal of the Autonomic Nervous System, 7, 279-294.

McCabe, P. M., Yongue, B. G., Ackles, P. K., \& Porges, S. W. (1985). Changes in heart period, heart period variability, and a spectral analysis estimate of respiratory sinus arrhythmia in response to pharmacological manipulations of the baroreceptor reflex in cats. Psychophysiology, 22, 195-203.

Overman, W. H., \& Doty, R. W. (1982). Hemispheric specialization displayed by man but not macaques for analysis of faces. Neuropsychologia, 20, 113-128.

Porges, S. W., McCabe, P. M., \& Yongue, B. G. (1982). Respiratory heart rate interactions: Psychophysiological implications for pathophysiology and behavior. In J. T. Cacioppo \& R. E. Petty (Eds.), Perspectives in cardiovascular psychophysiology (pp. 223-264). New York: Guilford.

Rosenfeld, S. A., \& Van Hoesen, G. (1979). Face recognition in the rhesus monkey. Neuropsychologia, 17, 503-509.

Sackett, G. (1965). Responses of rhesus monkeys to social stimulation presented by means of colored slides. Perceptual and Motor Skills, 20, 1027-1028.

Sackett, G. (1966). Monkeys reared in isolation with pictures as visual stimuli: Evidence for an innate releasing mechanism. Science, 154, 1468-1473.

Sackett, G., Griffin, G. A., Pratt, C., Joslyn, W. D., \& Ruppenthal, G. (1967). Mother-infant and adult female choice behavior in rhesus monkeys after various rearing experiences. Journal of Comparative and Physiological Psychology, 63, 376-381. 
Siddle, D. (1983). Orienting and habituation: Perspectives in human research. New York: Wiley.

Smuts, B. B., Cheney, D. W., Wrangham, R. W., \& Struhsaker, T. T. (1987). Primate societies. Chicago: University of Chicago Press.

Sokolov, Y. N. (1963). Perception and the conditioned reflex. New York: Pergamon.

Tranel, D., Fowles, D. C., \& Damasio, A. R. (1985). Electrodermal discrimination of familiar and unfamiliar faces: A methodology. Psychophysiology, 22, 403-408.

Turpin, G. (1983). Unconditioned reflexes and the autonomic nervous system. In D. Siddle (Ed.), Orienting and habituation: Perspectives in human research (pp. 1-69) New York: Wiley.

Turpin, G. (1986) Effects of stimulus intensity on autonomic responding: The problem of differentiating orienting and defensive reflexes. Psychophysiology, 23, 1-14.

Weisbard, C., \& Graham, F. K. (1971). Heart-rate change as a component of the orienting response in monkeys. Journal of Comparative and Physiological Psychology, 76, 74-83. 